\title{
Optimization of fractional composition of the excipient in the elastomeric covering for asphalt highways
}

\author{
E. M. Nurullaev, A. S. Ermilov \\ Perm National Research Polytechnic University. 614990 Perm, Komsomol prospect, 29.
}

\begin{abstract}
The computational method of optimum fractional composition of a dispersible filler of polymeric composite on the basis of three-dimensionally linked elastomer is developed according to non-linear programming. The coefficient of dynamic viscosity of polymeric suspension or the initial module of a viscoelasticity of the join solidification low-molecular rubbers with the final functional groups, filled by many fractional dioxide of silicon are considered as criteria of optimization. Influence of the limiting volume filling on energy of mechanical destruction was investigated. The elastomeric material is offered for use as a covering of asphalt highways in the form of a frost-proof waterproofing layer, which allowing multiply to increase operating properties.
\end{abstract}

KEYwORDS. Viscosity; Mechanical destruction; Elastomeric composites with a dispersed filler; Rheology; Rubber; Polymeric binder; Asphalt highways.

\section{INTRODUCTION}

$\mathrm{F}$ ractional composition of a dispersed filler is essential for formation of rheological behavior of suspensions on the basis of fluid and viscid polymeric binding and mechanical characteristics of three-dimensionally linked filled elastomers. At the same time the major parameter of composition is effective extent of volume filling $-\varphi / \varphi_{m}$, herein $\varphi$ - volume proportion of solids of an filler, $\varphi_{m}$ - the limiting extent of volume filling depending on a form of particles and their distribution by the size, and also from physical and chemical interaction on border "a filler - a binder". Value $\varphi_{m}$ can be defined by the viscometric method [1] or calculated by a combinatorial and multiplicative method [2].

Coefficient of dynamic viscosity $\eta$ and the initial module of a viscoelasticity $\mathrm{E}=\frac{\mathrm{d} \sigma}{\mathrm{d} \alpha},($ with $\alpha=1)$ are connected by a ratio (1):

$$
\eta_{r}=\frac{\eta_{f}}{\eta_{o}}=E_{r}=\frac{E_{f}}{E_{o}}=\left(1+1.25 \frac{\varphi / \varphi_{m}}{1-\varphi / \varphi_{m}}\right)^{2}
$$

herein the " $\mathrm{f}$ " and "o" indexes fall into to the filled and free conditions of the polymeric binding.

Energy (work) of destruction was estimated in the form of the envelope by curve of destruction [4] dependences of the conditional ultimate break tension $\sigma_{b}$ (tension divided by the initial section of the sample) from break deformation $\varepsilon_{b}$, bound to degree of the relative elongation by ratio $\alpha_{b}=1+\varepsilon_{b} / 100 \%$. The line envelopes of around points of a break of exemplars and constructed in $\log$ arithmic scale $\left(\log \sigma_{b}-\log \varepsilon_{b}\right)$, corresponds to energy of mechanical destruction in the form of the area of the chart of stretching in Cartesian coordinates: 


$$
A_{b}=\int_{1}^{\sigma_{b}} \sigma(\alpha) d \alpha
$$

herein structural and mechanical dependence of the conditional tension $\sigma$ from elongations extent $\alpha$ on condition of lack by abruption of particles of an filler from elastomeric binder, (for example, covering for asphalt), is proved by us earlier [5]:

$$
\sigma(\alpha)=v_{c b} \varphi_{r}^{1 / 3} R T_{\infty}\left\{1+29 \exp \left[-0.225 \cdot 10^{-3}\left(T-T_{\infty}\right)^{2}\right]\right\} \alpha^{-1} a_{\dot{\alpha}}^{-1}\left(1+1.25 \frac{\varphi / \varphi_{m}}{1-\varphi / \varphi_{m}}\right)^{2}\left(\alpha-\alpha^{-2}\right)
$$

herein:

$v_{c b}=\rho / \bar{M}_{c}:$ molar concentration of transversal chemical bonds in a polymeric basis a binder $\left(\rho-\right.$ polymer density, $\bar{M}_{c}$ - an average statistical molecular mass);

$\varphi_{r}$ : volume ratio of polymer in binder, containing softener;

$R$ : universal gas constant;

$T_{\infty}$ : equilibrium temperature at which concentration of transversal "physical" (intermolecular) communications $v_{p h}$ is negligible;

$T$ : test temperature of an exemplar;

$T_{g}$ : temperature of a structural glass transition of the polymeric binding;

$a_{\dot{\alpha}}$ : coefficient of high-speed mixing $\left(a_{\dot{\alpha}}=1, \dot{\alpha}=1.4 \cdot 10^{-3} s^{-1}\right.$-standart for applications);

$\phi:$ volume ratio of a dispersible filler;

$\varphi_{m}$ : the limiting extent of volume filling of the elastomers, depending on a form and fractional composition of particles, and also from physical and chemical interaction on border "a filler - a binder".

Value $\varphi_{m}$ can be defined by the viscometric method [1] or calculated by a combinatorial and multiplicative method [2].

Value $\alpha_{b}$ in the Eq. (2), as well as $\varepsilon_{b}$, searched with the help of Eq. (4):

$$
\alpha_{b}^{f}=\alpha_{b}^{o}\left(1-\sqrt[3]{\varphi / \varphi_{m}}\right)+\sqrt[3]{\varphi / \varphi_{m}} ; \quad \varepsilon_{b}^{f}=\varepsilon_{b}^{o}\left(1-\sqrt[3]{\varphi / \varphi_{m}}\right)
$$

herein the " $\mathrm{f}$ " and "o" indexes fall into to the filled and free conditions of an elastomer.

Breaking deformation of a elastomeric binder $\varepsilon_{b}^{0}$, defining by efficiency concentration of cross-links $\left(v_{e f f}=v_{c b}+v_{p b}\right)$, was set experimentally [5].

Research objective were development of a method of optimization of fractional composition of a dispersed filler for creation of a frost-proof waterproof elastomeric materials for covering for asphalt highways located in zones with sharply continental climate in form of rolled.

\section{THEORETICAL STUDY}

he problem of optimization of fractional composition of dispersible components of a polymeric material (for the given weight average particle sizes of fractions) taking into account realization of a condition of an optimality on other production characteristics can be formulated in the form of the formulation of a non-linear programming:

$$
\begin{aligned}
& \varphi_{m}(\vec{\varphi}, \vec{q}, \vec{d}) \Rightarrow \max ; \eta_{r} \Rightarrow \min ; E_{r} \Rightarrow \min \\
& \varphi_{j}^{o p t}=\varphi_{j 1}+\varphi_{j 2}+\varphi_{j 3}+\ldots+\varphi_{j m_{j}}=\sum_{v=1}^{m_{j}} \varphi_{j v} \\
& 0 \leq \varphi_{j v}^{\min } \leq \varphi_{j v} \leq \varphi_{j v}^{\max } \leq 1, v=1,2,3, \ldots, m_{j} \text { with } \forall_{j} \in I_{n} \\
& \varphi_{j}^{o p t}=\frac{x_{j}^{o p t} / \gamma_{j}}{\sum_{j \in I_{n}} x_{j}^{o p t} / \gamma_{j}}
\end{aligned}
$$


herein

$\vec{\varphi}, \vec{q}, \vec{d}$ : vectors of volume fractions, sponginess and particle sizes of fractions of dispersible components as a part of a polymeric material respectively;

$\varphi_{j}^{o p t}$ : optimum volume fractions of fraction an filler in structure;

$\varphi_{j v}:$ volume fraction

$v:$ fraction by $j$-type an filler in structure;

$m_{j}:$ number of $j$-type fractions of a dispersed component;

$\varphi_{j v}^{\min }, \varphi_{j v}^{\max }$ : respectively the lower and upper bounds for volume fractions of fractions of solid components in structure;

$x_{j}^{o p t}$ : optimum for the corresponding block of characteristics, for example, mechanical, mass concentration of firm disperse components in polymeric composition;

$\gamma_{j}$ : density of disperse components;

$I_{n}$ : a set of the indexes belonging to types of a filler, entering into a compounding of a polymeric material.

In view of complexity, the task includes restrictions such as equalities, will be transformed to a problem of nonlinear programming with restrictions such as inequalities. The quantity of independent optimized variables is equal $n=\left(m_{j}\right)-m$, herein

$m$ : number of types of solid components by a polymeric material.

Thus normalizing ratio in case of the solution of a task is carried out automatically.

$$
\sum_{j \in I_{n}} \sum_{\nu=1}^{m_{j}} \varphi_{j v}=\sum_{j \in I_{n}} \varphi_{j}^{o p t}=1
$$

Further the vector of optimum volume fractions of fractions of filler in composition is defined:

$$
\vec{\varphi}^{o p t}=\left(\varphi_{j v}^{o p t} ; \forall I_{j} \in I_{n}\right) ; \quad v=1,2,3, \ldots, m_{j}
$$

herein

$\varphi_{j v}^{o p t}:$ an optimum volume fraction

$v$ : fraction by $j$-type a filler.

Transition to optimum mass concentration of the relevant fractions of firm components

$$
\vec{x}_{j}^{o p t}=\left(\vec{x}_{j v}^{o p t} ; \forall{ }_{j} \in I_{n} ; v=1,2,3, \ldots, m_{j}\right)
$$

is carried out on a formula: $x_{j v}^{o p t}=\left(\phi_{j v}^{o p t} \gamma_{j}\right) /\left(\phi_{j}^{o p t} / \gamma_{j}\right) \cdot P$

herein $P=\sum_{j \in I_{n}} \sum_{\nu=1}^{m_{j}} x_{j v}^{o p t}=\sum_{j \in I_{n}} x_{j}^{o p t}$ is the sum of mass concentration (shares) of solid components by a polymeric compositing.

\section{EXPERIMENTAL STUDY}

C haracteristics used a fraction of silica as a dispersed filler for slurry on based low-molecular rubbers (oligomers) with final epoxy groups polydienurethanepoxide (trademark PDI-3B) and carboxyl groups polybutadiencarbocsilate (trademark SKD-CTR) are shown in Tab. 1.

Surface by response of function in the projection in the chart Gibbs "composition-property" (Fig. 1), which we obtained by the developed computer program [3], demonstrates the calculated dependence of value $\eta_{r}=\eta_{f} / \eta_{o}$ the investigated polymer slurry from the volume ratio of the three fractions of silica, differing characteristics in accordance with Tab. 1. Constant volume fraction of filler is 0.75 . In all cases, the calculation of the limit of bulk fill $\varphi_{m}$, through the coefficients of the porosity of various mixtures of fractions implemented with help by the coefficients of the porosity of individual fractions identified viscometric method [1]. At the same time physical and chemical factors influencing the limiting filling 
of a polymeric binder takes into account "automatically", according to the physical nature of viscosimetric method. It can be seen that the minimum value of the relative coefficient dynamic viscosity $\eta_{r}$ is provided an optimal ratio of volume fractions of fractions (rounded): $600 \mu 30 \mu 1 \mu=0.5: 0.3: 0.2$. It shows also the experimental values $\eta_{r}$.

\begin{tabular}{cccc}
\hline $\begin{array}{c}\text { Characteristics } \\
\text { Porosity }\end{array}$ & 1 (small) & 2 (medium) & 3 (large) \\
$\begin{array}{c}\text { (volume fraction of pores) } \\
\begin{array}{c}\text { Void ratio } \\
\text { (the ratio of the volume fractions } \\
\quad \text { of pores and particles) }\end{array}\end{array}$ & 0.450 & 0.384 & 0.379 \\
Weight average particle size, $\mu$ & 1 & 0.618 & 0.610 \\
\hline
\end{tabular}

Table 1: Characteristics fractions of silica.

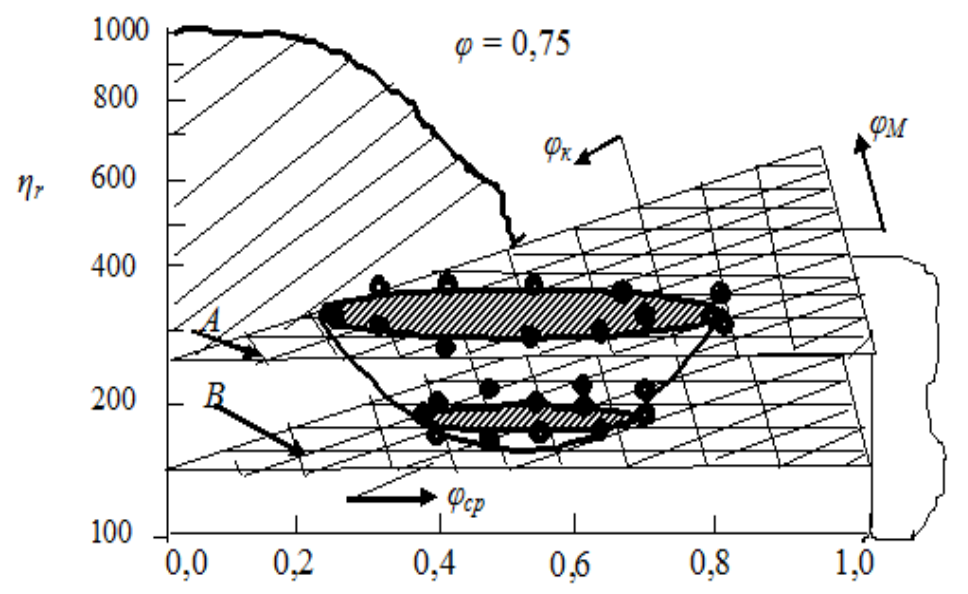

Figure 1: The calculated and experimental dependences of the relative dynamic viscosity polymer slurry based by polydinurethanepoxide (PDI-3B) and polybutadiencarboxilate (SKD-CTR) from volumetric ratios of 3 fractions of silica: A, B - levels calculated iso-viscosity, round icons - experimental data.

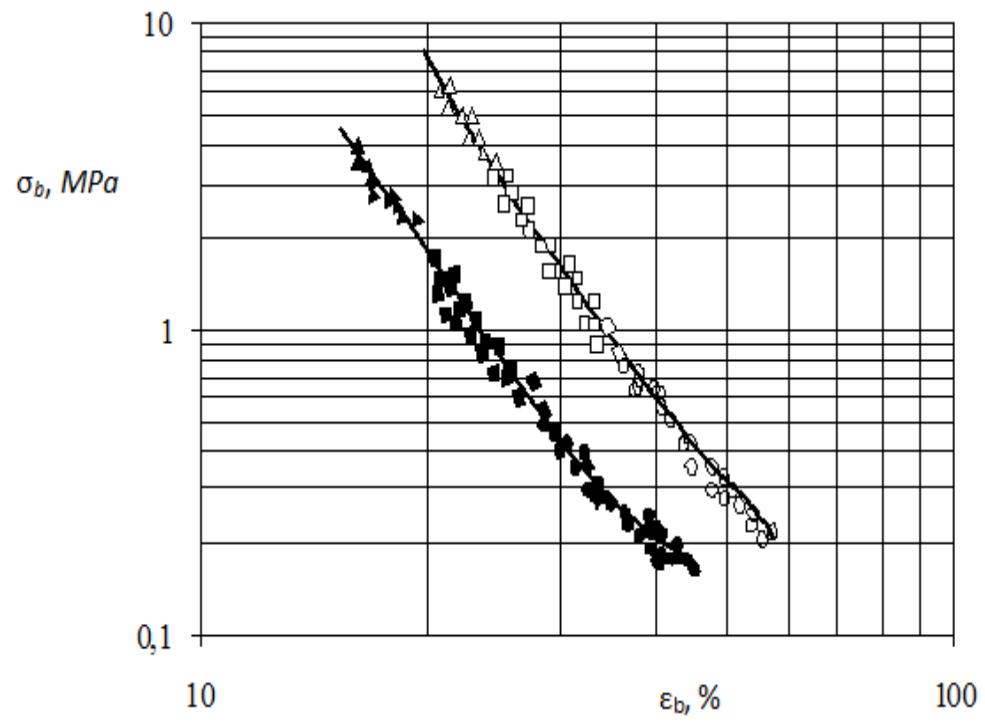

Figure 2: Envelopes by points of destruction $\left[\sigma_{b}(M P a)=f[\varepsilon b(\%)]\right.$ (in logarithmic scale) of the samples elastomer filled bifraction (black symbols) silica three fraction (white symbols) of silica:

$\mathrm{O}$ a sample of $\mathrm{T}=323 \mathrm{~K}$

$\square \quad$ a sample of $\mathrm{T}=223 \mathrm{~K}$

- the standard $\mathrm{T}=323 \mathrm{~K}$

$\triangle$ a sample of $\mathrm{T}=293 \mathrm{~K}$

- the standard $\mathrm{T}=293 \mathrm{~K}$
the standard $\mathrm{T}=223 \mathrm{~K}$ 
Experimental study of prescription's parameter $\varphi / \varphi_{m}$ filled elastomer at strain-strength characteristics, expressed in the form of envelopes by destruction was carried out using silica the following fractional composition:

1 - initial test sample - (240:5) $\mu=(20: 80) \%$

2 - prototype - (240: 5: 1) $\mu=(40: 40: 20 \%)$.

As the polymer binder used stoichiometric mixture (1: 2 moles) of low molecular weight rubber trademarks PDI-3B and SKD-CTR, three-dimensionally cross-linked three-functional aromatic epoxy resin trademark EET-1 (1 mol). Specific surface area of contact "filler-binder" in both cases remained constant.

Indication in Fig. 2 (with the normal motion from the lower to the upper right) shows that at a fixed value of the volume fraction of silica $\varphi=0.712$ modified effective volumetric filling $\varphi / \varphi_{m}$ from $0.712 / 0.752=0.946$ to $0.712 / 0.816=0.72$ leads to an increase in the energy by mechanical destruction by elastomeric composite 1.5 - 1.7 times. Reducing the maximum degree of volume filling from 0.946 to 0.872 promotes increase "return" of the polymeric binder in the growth of energy mechanical destruction elastomer composite in accordance with the mathematical relationship (2, 3, 4). [5].

Thus, the use by optimal multifractional filler for constant chemical composition of the composition allows for increase significantly in service life of the studied composite material which offered as frost-resistant waterproof covering (oilfired sub-layer) for asphalt highways located in areas with sharply continental climate.

Use as frost-resistant waterproofing covering which filled three-dimensional cross-linked elastomer, provides an elastically deformability surface of the road-load transport in the temperature range of $223 \mathrm{~K} \ldots 323 \mathrm{~K}\left(-50 \ldots+50^{\circ} \mathrm{C}\right)$; this prevents the massive destruction of asphalt at alternating temperatures and operating loads at the expense of phase transitions' water-ice-water ", which accompanied by a volume expansion of ice when water freezes in the initial cracks of asphalt.

The following is an example of the engineering realization of recommended material.

$\checkmark$ The composition contains: A polymer binder (in the ratio 1: 2) polydienurethanepoxide with final epoxy groups (PDI-3B) and polybutadiencarbocsilate with a terminal carboxyl groups (SKD-KTR) (13.5 wt. \%),

$\checkmark$ Natural macro crystalline quartz (57.4 wt.\%); Fumed silica grade "Aerosil-380" (24.6 wt.\%),

$\checkmark$ Processing aids: thixotropic amplifier of elastomer and pigment - technical carbon (3.0 wt.\%), three-dimensional cross-linking agent - epoxy gum brand EET-1 (1.45 wt.\%), three-dimensional crosslinking catalyst - acetolacetonate Fe $(0,05$ wt. $\%)$.

$\checkmark$ Blending components conducted at $55-60^{\circ} \mathrm{C}$ in a continuous mixer such as "SND-75", followed by formation of canvas width 3.0 meters and a thickness of $0.012 \mathrm{~m}(12 \mathrm{~mm})$. Three-dimensional cross-linking the polymer base material was conducted out in a continuous drum-vulcanizer at a temperature $170-180{ }^{\circ} \mathrm{C}$ with a residence time in the apparatus 7-5 minutes respectively. received and rolled from rolls ( 25 meters long each) on the asphalt, smearing by liquid bitumen (oil), waterproof canvas with a relative speed of uniaxial tension $1.4 \cdot 10^{-3} \mathrm{~s}^{-1}$ had the following mechanical characteristics specified in Tab. 2. In the same part of the recommended composition based on a threedimensional cross-linked elastomer filled three-fraction silica.

Such a roll material on intermediate "glue" layer of liquid bitumen (oil), is to protect the asphalt canvas based on bituminous binder, becoming brittle in the cold, from the ravages of a pair of "water - ice" These characteristics indicate on increased frost-resistant developed coating of asphalt highways and you can use it in a wide temperature range for 20 ... 30 years.

\begin{tabular}{|c|c|c|c|c|c|c|c|}
\hline \multirow{2}{*}{ Composition } & \multirow{3}{*}{$\begin{array}{l}\text { Volume } \\
\text { fractions }\end{array}$} & \multicolumn{6}{|c|}{ Mechanical properties } \\
\hline & & \multicolumn{2}{|c|}{$323 K$} & \multicolumn{2}{|c|}{$293 K$} & \multicolumn{2}{|c|}{$223 K$} \\
\hline \multirow{3}{*}{$\begin{array}{l}\text { Polimeric binder: } \\
\text { Rubber SKD-CTR; } \\
\text { Rubber PDI-3B; } \\
\text { Epoxy EET-1 }\end{array}$} & & $\sigma_{b}, M P a$ & $\varepsilon_{b}, \%$ & $\sigma_{b}, M P a$ & $\varepsilon_{b}, \%$ & $\sigma_{b}, M P a$ & $\varepsilon_{b}, \%$ \\
\hline & \multirow[b]{2}{*}{0.288} & 0.25 & 55 & 1.20 & 32 & 6.00 & 22 \\
\hline & & & & & & & \\
\hline $\begin{array}{c}\text { Filler: } \\
\text { Silica } \\
(240: 5: 1) \mu=(40: 40: 20) \%\end{array}$ & 0.712 & & & & & & \\
\hline
\end{tabular}

Table 2: Mechanical characteristics of composition. 


\section{CONCLUSIONS}

$\mathrm{B}$

ased on non-linear programming was developed mathematical optimization method of fractional composition of the disperse filler, which significantly affects the coefficient of dynamic viscosity of the suspension and the energy of mechanical destruction three dimensional cross-linked elastomeric composite. Also was proposed formulation of the polymer compositions based on low molecular rubbers with final functional groups, filled with silica optimal fractional composition, allowing increase the service life of asphalt highways. In relation of frost-resistance and waterproofing of the created coating based of the filled elastomer, engineering example shows the practical effectiveness of the proposed method to increase the service life of asphalt highways.

\section{REFERENCES}

[1] A. S. Ermilov, K. Zyryanov, Plant Laboratory. Diagnostic materials, 67(9) (2001) 62.

[2] A. S. Ermilov, A. M. Fedoseev, J. of Applied Chemistry, 77(7) (2004) 1218.

[3] Certificate number 2012613349 RF. software identify and optimize the packing density of solid dispersed polymer composite fillers materials (rheology). / Ermilov A. S, Nurullaev E. M., Duregin K. A. - Priority from 09.04.2012.

[4] T. L. Smith, J. Appl. Phys., 35 (1964) 27.

[5] A. S. Ermilov, E. M. Nurullaev, Mechanic composite materials, 48(3) (2012) 359. 